\title{
CQI Classification Based Method to Improve Throughput in Wireless LTE Systems
}

\author{
Suganya Srinivasan ${ }^{1}$ and Ramesh ${ }^{2}$ \\ ${ }^{1}$ Research Scholar \\ ${ }^{1,2}$ VTU, Belagavi, India \\ ${ }^{1}$ suganya.senthil2005@gmail.com
}

\begin{abstract}
Advanced wireless networks such as $4 G$ LTE have effective ways of reporting channel quality through CQI values of the time and frequency varying channel whether it is fast fading or slow fading. The main objective of using CQI values is to schedule the resource blocks and maintain proper SNR by adjusting signal power or modulation. In doing so, one must precisely be able to map CQI values to SNR values so that the objectives can be fulfilled properly where an imperfect mapping can hamper the performance of wireless system as packet scheduling can become difficult and unmanageable. In this work, a detailed study of variation of channel SNR is presented and a classification based method for scheduling packets is proposed for varying SNR. The proposed method is shown to improve performance at link level and packet level throughout. Our future work is to create a fine grain system considering various channel models and incorporate same in the proposed model.
\end{abstract}

Keywords: LTE, Imperfect CQI, packet scheduling, fair queuing, proportional fair, channel allocation, resource blocks

\section{Introduction}

Future Wireless Networks such as LTE (Long Term Evolution) and LTE-A (LTEAdvanced) have novel ways to utilize frequency spectrum efficiently may it be using OFDM (Orthogonal Frequency Division Multiplexing) or using optimal channel and source coding. LTE also utilizes prediction resource allocation schemes including but not limited to Adaptive Modulation and Coding (AMC), Channel Quality Index (CQI) and Hybrid Automatic Retransmission Request (HARQ) as detailed in [1]. LTE system could make utilized for uplink (towards the resource allocator from UE) alternately as a downlink (from the resource allocator to the UE). In time division Multiplexing (TDM), the accessible bandwidth is time divided among end users same time in frequency division Multiplexing (FDM) same frequency groups are used to differentiate separate end users. Different end users might utilize the FDM at the same time utilizing FDMA (Frequency division different Access). In LTE and LTE-A, Orthogonal Frequency Division Multiple Access (OFDMA) is used to attain higher data information. For scheduling the resource, of LTE, RBs (Resource Blocks) are designed where user perform hop beyond certain RB (time or frequency block) after the subsequent Resource Block. Scheduling this hoping users among exceptional RBs a challenge so specific users have special QoS (Quality of Service), delay, throughput and bandwidth requirements. On one hand, LTE Release 8 is an outcome of research of many years in telecom industry which is based on OFDM techniques. The deployment of LTE is mostly considered as a prime contender for macro and micro cells providing improvised system capacity, coverage, data rate and latency as compared to its previous generations. LTE-A (advanced) on the

Received (April 20, 2017), Review Result (August 30, 2017), Accepted (September 8, 2017) 
other hand is an important improvement of LTE release 8 which enhances the user experience in high mobility situations [8]. The community has added up to Release 14 into the research domain for which is all collectively known as LTE-A after release 8. Wireless throughput is a term used to measure the performance of a wireless channel. Techniques to improve performance of a wireless channel not only include the coding, modulation and scheduling, but also require a deep understanding about the channel features such as multipath fading, slow and fast fading, and channel gain. Upon successfully analyzing these features, one can optimally design a scheme to schedule resource blocks (RBs) to provide better throughput. There are many ways of improving throughput of an LTE channel. Rate and delay feedback, channel gain equalization and diversity techniques are well known in literature. Moreover, OFDM provides a neat way to use single subcarrier in both time and frequency domain to transmit signal in a resource block. While it is known that techniques such as FHSS can be used, packet scheduling in a RB is still a major challenge as it requires a multi-criteria optimization methodology. Various packet scheduling schemes are proposed in the literature including proportional fair, round robin, fair queuing, weighted fair queuing, inverse weighted queuing and inverse weighted fair queuing [2-5]. In order to create a better scheduling, focus is provided on different aspects of a channel such as number of subcarriers, number of resource blocks, quality of channel, modulation scheme used e.g. Most of the work in literature focus on specifics of parameters as mentioned above. While this approach is useful in many cases, the results can be improved if we can provide a holistic approach which can focus on group based usage of scheduling [9-10].The advantage of classification and grouping are as follows: (a) One can be characterized as a part of group, (b) a group level guaranteed QoS can be maintained, (c) There is a lower computation complexity at the downlink transmitter and computing resources and (d) Estimates of channel can be used directly to improvise the system as a whole. In this work, a CQI classification based method is proposed to improve throughput of a 4G LTE wireless system. The paper is organized as follows: Section II details various techniques of resource scheduling in LTE. Section III describes the importance of CQI and its usage in modern networks for better channel utilization. Section IV explains our proposed classification based method for packet scheduling. Results and analysis is presented in Section V while Section VI concludes the paper with briefs on our future work.

\section{LTE Resource Scheduling}

Every user is assigned various alleged resource blocks (RBs) in the time frequency framework. End user's bit rate relies on the quantity of RBs and the utilized modulation. The amount from claiming RBs and the sort of resources an end user gets allocated may be purely subject to those scheduling algorithm which we use in time and frequency recurrence measurements. In Figure 1 physical resource block (PRB) is the fundamental square or unit of LTE and the designation of all these PRBs depend on the scheduling methodology eNB. Each LTE sub-outline is of $1 \mathrm{~ms}$ term and comprises of two slots, with each space being $0.5 \mathrm{~ms}$. There are seven OFDM slots in the time space of each slot.

In control data or all the more particularly inside downlink control data, eNodeB sends the subtle elements of resources through which UE can collect its downlink information or UE can send their uplink information. Besides, the control data information is comprised of CCE's and genuine information data is made out of RBs. LTE has $20 \mathrm{MHz}$ data transmission band width and $100 \mathrm{RBs}$ [2]. 


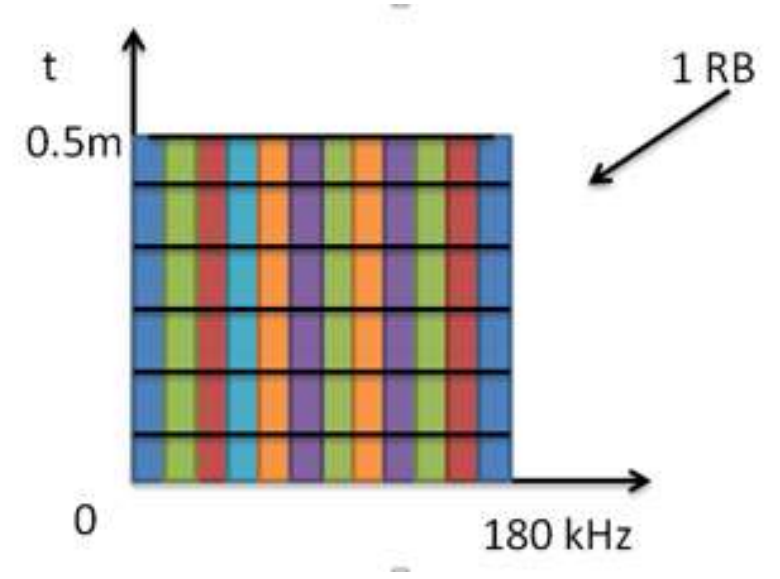

Figure 1. Schematic of one Block (RB)

On the off chance that eNB needs to allot just 20 RB's to UE, the data inside DCI will empower UE to figure correct RBs distributed to it. In case eNB needs to send 100 bits, the system will build control data information. To decrease the extent of control data, LTE presents diverse resource distribution methodologies.

Network congestion and instability problems are reduced by different resource scheduling methods. A plenty of methodologies are proposed for the segregation of resources among users which can be elaborated as follows: (1) channel unaware, (2) channel aware/QoS unaware, (3) Channel aware/QoS aware, (4) semi-persistent for VoIP support and (5) energy aware [1].

Initially, "Channel Unaware" systems are basically produced for wired correspondences wherein the transmission media is invariant regardless of the possibility that there is any adjustment in time. Likewise, the likelihood of error occurrence in a wired media is insignificant. In demonstrate hatred for that wired communications' suspicions don't function admirably in wireless situation, they shape a base for some practical and powerful scheduling strategies which can be explained as takes after:

(a) First In First Out: This is most straightforward calculation to distribute resources for UE's. The main demand will be served or will get the assets first. This is an inefficient calculation because of the way that meriting users don't get the resources.

(b) Round Robin: Here every single user will get resources in their designated time term, yet even this is not an effective technique as it prompts deferral of execution.

(c) Blind Equal Throughput (BET): This is a genuinely better strategy as it takes the past normal throughput of every user and based on the data received for every one of the users, it will plan the users with slightest normal throughput by sharing a few resources from the most elevated normal throughput user.

(d) Resource Preemption: Here it makes utilization of the priority techniques to allot the resources to the UE. The user with most astounding need experiences resource acquisition.

(e). Weighted Fair Queuing: To maintain a strategic distance from the starvation condition happened due to the need strategies, weighted reasonable lining is utilized. Here resources will be designated in view of the weights given.

(f). Guaranteed Delay: In this strategy an opportunity to live for every packet is assigned. The packet must touch base inside that specific due time or is disposed of.

Secondly, in "Channel aware/QoS unaware methodology", the channel condition assumes a vital part. The most extreme channel limit is administered by Shannon's principle. Given the transmission capacity and noise in the channel, the maximum information rate in the channel can be resolved. The algorithms can be recorded as takes after: 
(a) Maximum Throughput (MT): In the event that the user has the best channel condition, at that point that specific user will be allocated with most extreme throughput.

(b) Proportional fair (PF): Channel unaware BET can be utilized along with MT to dispense resources proportionately which enhances the range productivity and reasonableness in resource conveyance.

(c) Joint Time and Frequency space Schedulers: For this situation an arrangement of dynamic UEs are chosen by Time Domain Packet Schedulers (TDPS) and the portion of resources to those UEs is finished by the Frequency Domain Packet Scheduler (FDPS).

(d) Inverse weighted Fair Queuing (IWFQ): The network sections must be swapped so as to represent BET and thus the weights related with every user will be straightforwardly corresponding to throughput. In this way, in this calculation the resources are shared proportionately among users by taking inverse of their weights joined to it [2].

$$
y_{i, l}^{I W F Q}=\left(1 / w_{i}\right) \cdot y_{i, l}^{R R}
$$

(e) Proportional Inverse Weighted Fair Queuing (PIWFQ): PF, BET, IWFQ all these three methods combined together, gives PIWFQ to achieve fairness in the scheduling strategies [2].

$$
G_{i, l}^{P I W F Q}=G_{i, l}^{M T} \cdot G_{i, l}^{B E T} \cdot y_{i, l}^{I W F Q}=\frac{g_{l}^{i}(T)}{f^{i}(T-1)} \cdot \frac{1}{w_{i}} \cdot y_{i, l}^{R R}
$$

Thirdly, "Channel known/QoS known" methodologies have (a) Schedulers for Guaranteed information rate, (b) Schedulers for Guaranteed defer prerequisites and (c) Dynamic Schedulers for VoIP. Fourthly, "Semi-Persistent Scheduling for VoIP" does not contribute for the ghostly productivity and it can be considered as like "channel uninformed" approach. The same can't be sent in remote conditions because of its inalienable impediments. At last, "Energy-aware" systems diminish the power utilization and enhance the spectral efficiency. A basic technique to diminish the power utilization is by changing the eNB to rest mode every now and again amid non-crest hours or utilizing measurement information examples of past.

Wireless channel is known to be noisy. A simple AWGN (Additive White Gaussian Noise) model suggests that we can consider received signal as the linear addition of transmitted signal with a random zero mean Gaussian noise. While this model significantly explains the behavior of a channel, it is does not consider the time variance and frequency variance (Doppler) of the channel. Also, the effect of multi-path is ignored while considering the received signal which is a complex function of noise and channel gain.

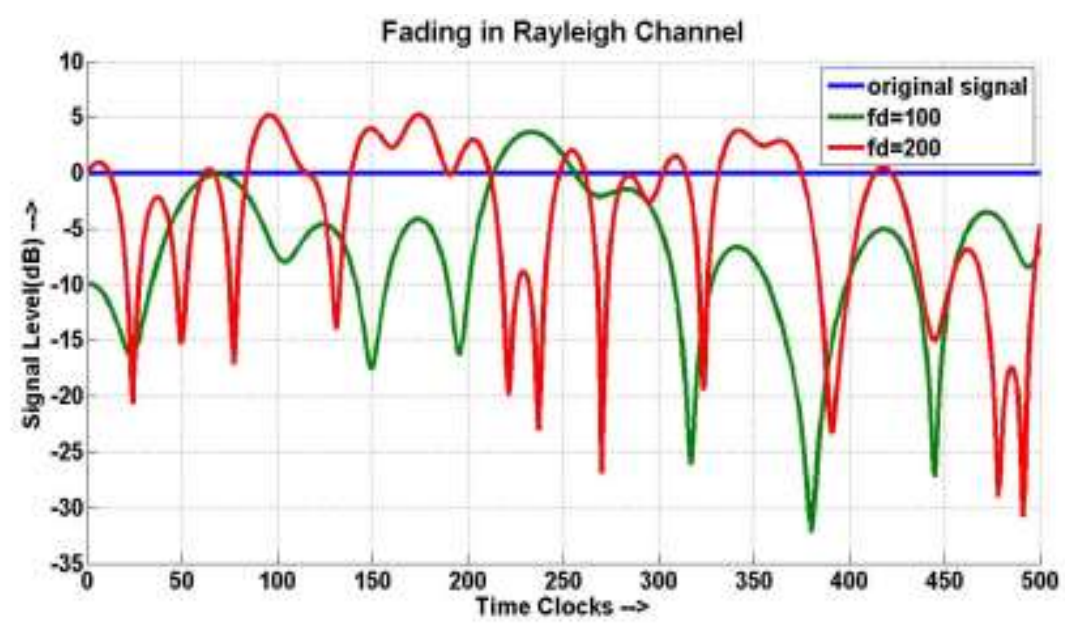

Figure 2. Fading in Rayleigh Channel for Different Doppler Frequency 
Figure 2 shows a Rayleigh faded signal with two different Doppler frequencies. A simple channel model can be explained using equation below where the received signal is a linear combination of: (a) x: transmitted signal, (b) h: channel gain and (c) n: Gaussian noise.

$$
\bar{y}=h x^{+} n
$$

While the signal is highly noisy and time varying, one can also find the correlation between two instances of a same signal and signal correlation with itself as shown in Figure 3. We can clearly observe that when the signal correlates itself at $\mathrm{fd}^{*}$ deltaT $=0.4$ that is after around every $\lambda / 2$ cycle. Therefore, one can take advantage of this property and receive correlated signal at the receiver every half cycle of a signal. This property is highly useful in determining the SNR (signal to noise ratio) of the signal at the transmitter and the receiver.

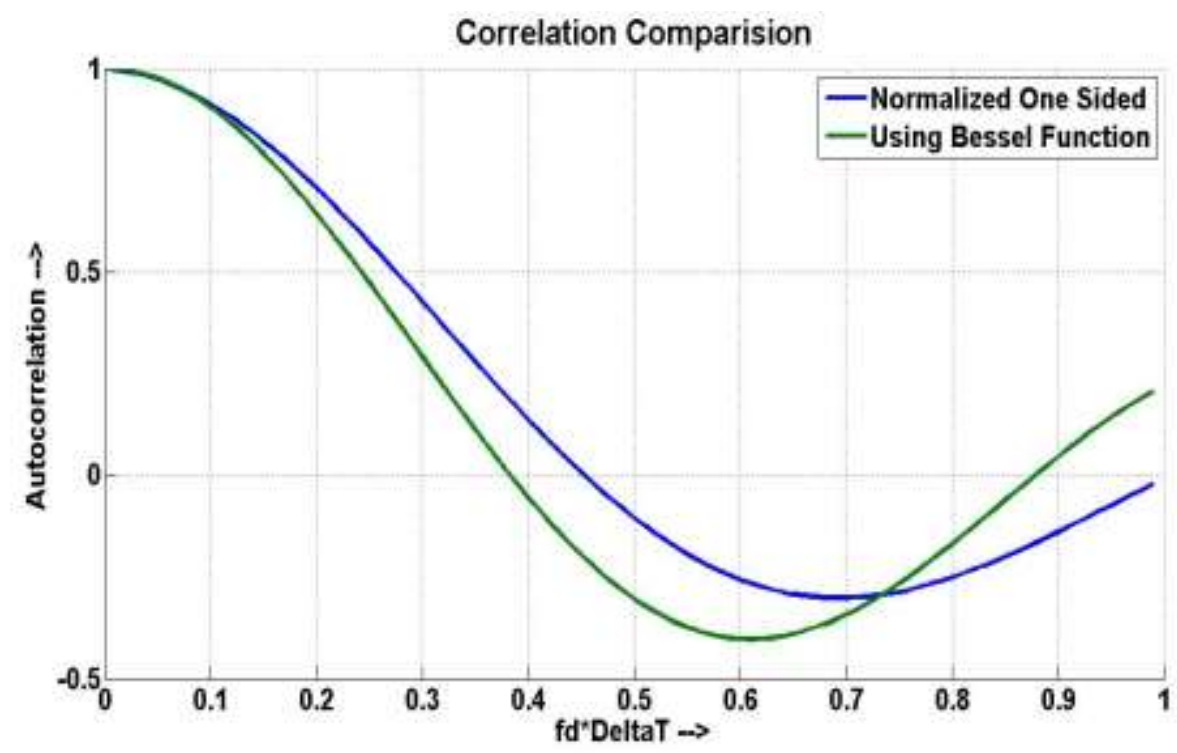

Figure 3. Correlation of a Faded Signal

The analysis has many advantages. For example, one can tune the receiver to receive only highly correlated signal and therefore to avoid highly noisy signal. Also the channel inversion can be used at the receiver to overcome the phase shift of the signal. These properties of a channel quality are highly important to understand in order to optimize LTE channel scheduling and therefore throughput as CQI (Channel Quality Indicator) values are provided in LTE to understand the quality of channel. Channel utilization and CQI are detailed in the next section.

\section{Channel Utilization and CQI}

A channel can be effectively utilized if one can use proper scheduling and modulation scheme so that the effect of channel noise and gain can be nullified at the receiver. LTE receiver periodically sends a CQI value to the transmitter so that transmitter can use good modulation scheme based upon channel state of transmitter. A CQI table is the group of 1-15 integers each denoting the type of modulation scheme to be used and other parameters pertaining to the channel. For instance $\mathrm{CQI}=5$ indicates QPSK modulation with MCS=5. A CQI value of 15 is used for 64 QAM in current LTE design. One can also estimate the SINR values from CQI values as detailed in [6]. The value of reported CQI value in a frequency selective scheduling mode may be inaccurate due to channel 
fluctuations and therefore one must verify its value by suitable pilot methods. The channel utilization can be written as a function of accuracy of CQI reporting as it directly impacts the modulation scheme and transmitted signal power.

$$
\text { Channel Utilization }(\mathrm{CU})=\text { function }(\mathrm{CQI})(1-\mathrm{p}(\mathrm{e}))
$$

Where $\mathrm{p}(\mathrm{e})$ is the probability of error in reporting CQI. As $\mathrm{p}(\mathrm{e})$ is dependent upon fluctuations in the channel (Rayleigh channel is this case), we may argue that $p(e)$ is a Rayleigh distributed random variable with whose probability density function (pdf) can be written as:

$$
y=\frac{x}{b^{2}} \exp \left(-x^{2} / 2 b^{2}\right)
$$

In case of a slow faded flat channel, we can assume that the CQI value do not change much frequently. The problem arises in case of a fast fading channel wherein the SNR values change much frequently and therefore there might also be error in reporting CQI to the receiver. Figure 4 illustrates the channel utilization of a Rayleigh faded channel using the appropriate CQI value. We can clearly see that the channel is utilized very poorly in at many a times. In order to overcome this fluctuation, a method is proposed in the next section of this paper.

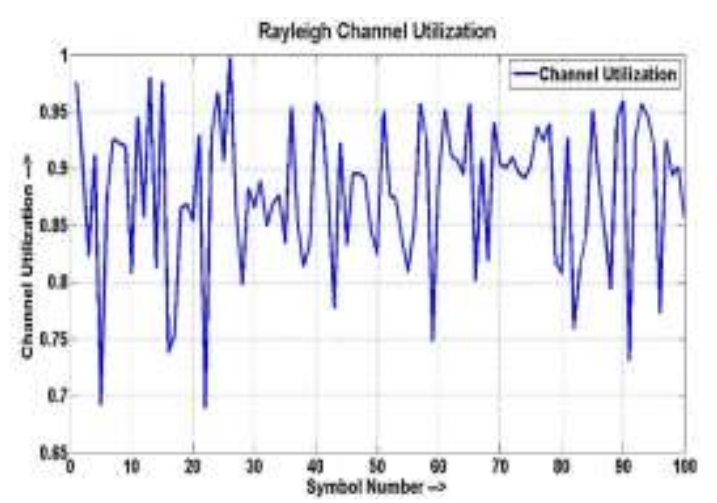

Figure 4. Channel Utilization for a Rayleigh Faded Signal

\section{Proposed Method}

In order to overcome the situation of imperfect CQI we can use Forward Error Correction (FEC) which requires previous levels of CQI [9]. In FEC approach, we can use levels of CQI using a Group Based Moving Average (GBMA) technique as shown in Figure 5. We arrange users in view of their evaluated CQI levels and pick the proper packet scheduling algorithm for each gathering. Once the SNR levels are calculated, we can choose the calculation use for various gathering of users. The decision is sensible in light of the fact that when the clients who are as of now at most noteworthy SNR levels can simply be given resources proportionately and they would have the capacity to get great throughput owing their better channel conditions when contrasted with the ones who are battling with resources and in addition with the channel conditions. We utilize the GBMA technique for allotting diverse booking calculation according to the value of user gathering. The scheduling strategies utilized are Maximum Throughput (MT), Proportional Fair (PF), Inverse Weighted Fair Queuing (IWFQ) and Proportional Inverse Weighted Fair Queuing (PIWFQ). 


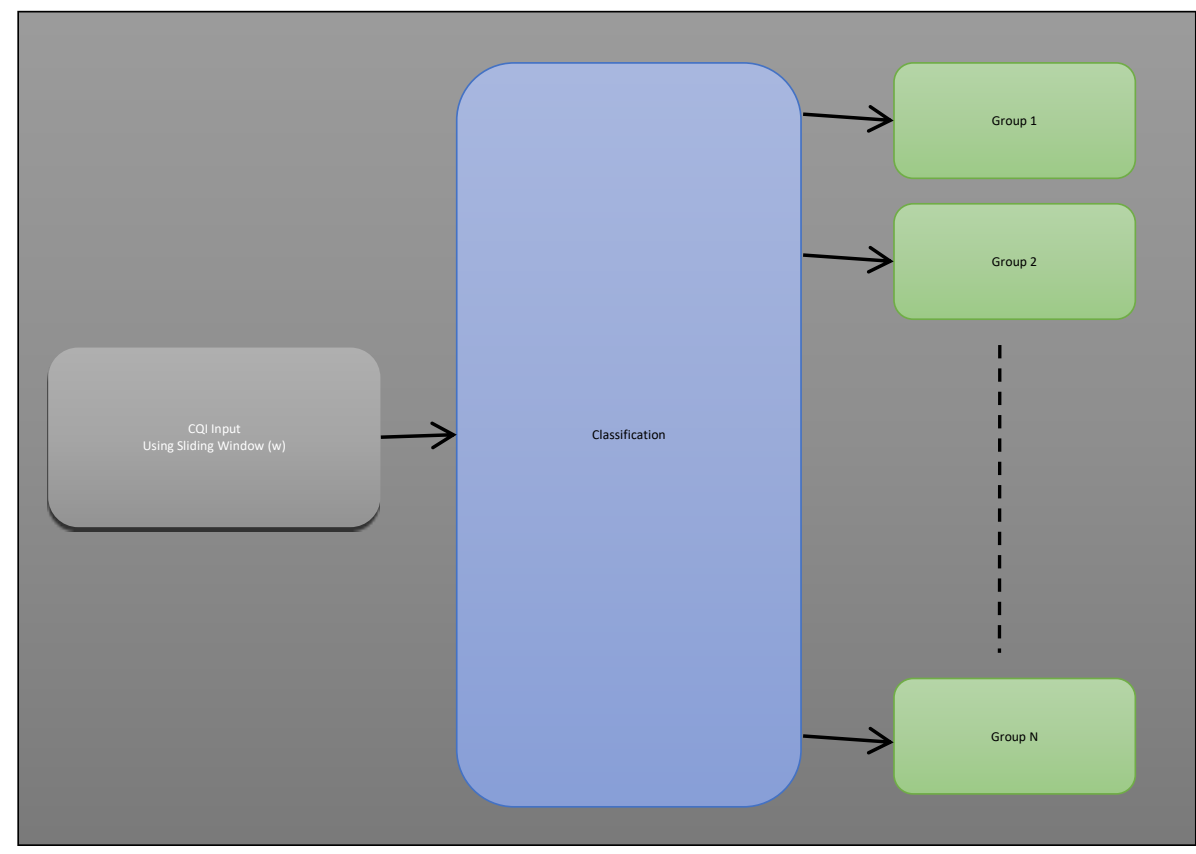

Figure 5. Channel Utilization for a Rayleigh Faded Signal

On the off chance that, we consider the need of premium users, we can rapidly improve the technique by utilizing a pre-settled measure of drifting transfer speed for need users which can be allotted to others when not being used. In this way, the planning won't be influenced for others. There might likewise be situation when the user bunches are unique for quite a while and for this situation the general normal throughput will be low for the system. We have similarly separated the sizes of SNR to allot the required planning calculation. The same can likewise be proportionately partitioned and is a zone left for investigation in future. This framework intensely depends upon the blemished CQI levels which are a typical situation in wireless channels.

The method works as follows:

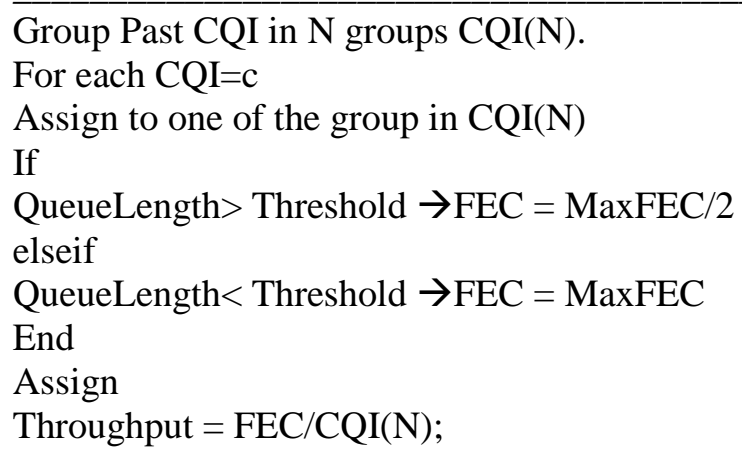

Here we can claim that for a group of users in better channel conditions, we can assign lower throughput as the losses will be minimum. On the other hand, for the users with bad channel conditions, we pump in more bits in the channel to provide them with minimum rate as required for the survival of service and hence the Quality of Service (QoS). Therefore, if channel condition is good, the number of FEC frames inserted reduced and vice versa. It is also observed while simulations that there is a need of fairness in order to deal with the system level optimization. In 
the group based method, the fairness is improved on a group level and therefore is appropriate to say that a better group may provide better throughput.

\section{Results and Analysis}

We use MATLAB and its channel tools for simulation in this paper. The results are shown for $\mathrm{CQI}=1: 15$ and group based method. Following Figure 6 shows the throughput improvement in case of proposed method as compared to the baseline version which is without using groups. We can observe that the throughput is improved for some user while it is lower for some users as compared to the baseline version. Those are users who are in good channel conditions and therefore the advantage can be taken of this fact. Also, we can say that the proposed method provides more fairness as compared to the existing scheme.

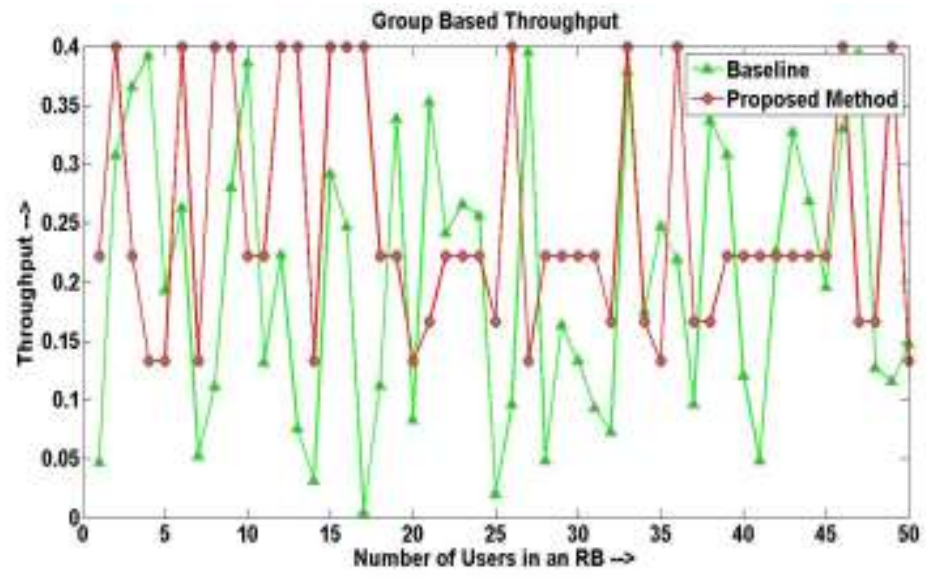

Figure 6. Throughput for Different Users

Figure7 compares the average throughput for different number of users taking advantage of the proposed scheme. It can be observed that even though the individual user might be hampered from the scheme (Figure 6), the overall throughput is higher as expected for a group as we treated the group as a whole to provide throughput. Table I details various parameters used in the simulations. In cases where comparisons are made, a higher or lower value of the parameter is used to compare when deemed appropriate. It can be noted that appropriate values of frequencies are used in Section II to compare the Rayleigh fading. Also, the simulations are run for a minimum of 50 times and average is taken in the all the cases to avoid the impact of any randomness occurred due to software itself.

Table I. Simulation Parameters

\begin{tabular}{|c|c|}
\hline Parameter & Value \\
\hline Channel & Rayleigh \\
\hline Number of RBs & 25 \\
\hline \#Users in RBs & 50 \\
\hline TTI & $1 \mathrm{~ms}$ \\
\hline Max Throughput & $100 \mathrm{kbps}$ \\
\hline Mode of Operation & FDD \\
\hline
\end{tabular}




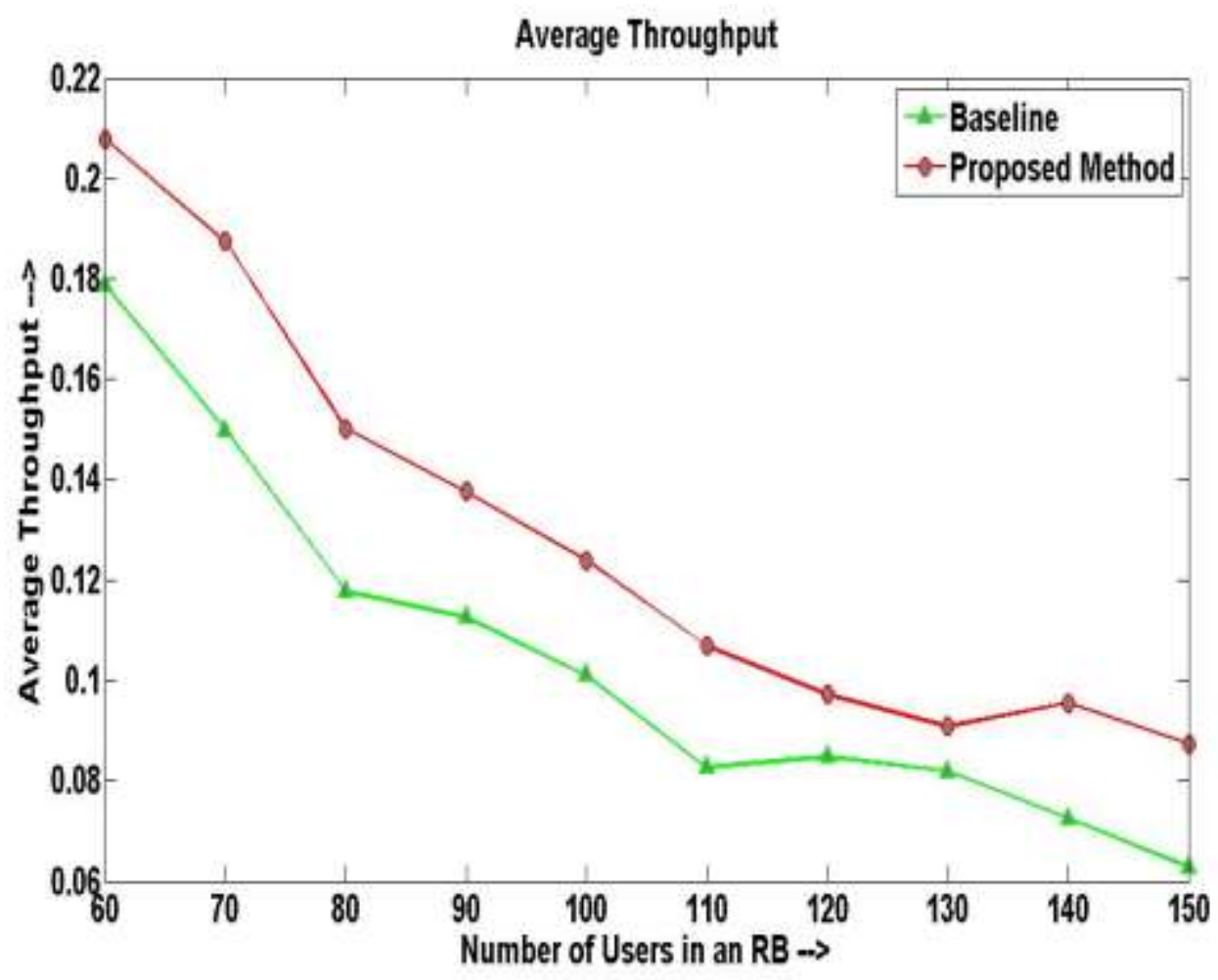

Figure 7. Average Throughput for Different Users

\section{Conclusion and Future Work}

In this paper, a detailed study of variation of channel SNR is presented and a classification based method for scheduling packets is proposed for varying SNR. In general, the Rayleigh channel is analyzed and it is observed that inherent advantages of multipath fading can be used to improve the system in total. The proposed method is shown to improve performance packet level throughout while it is suggested to use the channel gains to improve the link level throughput. Our future work is to create a fine grain system considering various channel models and incorporate same in the proposed scheme.

\section{References}

[1] F. Capozzi, "Downlink packet scheduling in LTE cellular networks: Key design issues and a survey", IEEE Communications Surveys \& Tutorials, vol. 15, no. 2, (2013), pp. 678-700

[2] S. Suganya, M. Sumit, S. L. Yaram and C. Ramesh, "Resource Scheduling Algorithms for LTE using Weights", in 2nd International conference on Applied and Theoretical Computing and Communication Technology, SJBIT, Bangalore, (2013).

[3] Phunchongharn, Phond, E. Hossain and D.I. Kim, "Resource allocation for device-to-device communications underlaying LTE-advanced networks", IEEE Wireless Communications, vol. 20, no. 4, (2013), pp. 911.-100.

[4] M. Sumit, "A joint parametric prediction model for wireless internet traffic using Hidden Markov Model", Wireless networks, vol. 19, no. 6, (2013), pp. 1171-1185.

[5] R. Kwan, C. Leung and J. Zhang, "Proportional fair multiuser scheduling in LTE", IEEE Signal Processing Letters, vol. 16, no. 6, (2009), pp. 461-464.

[6] G. Piro, "Two-level downlink scheduling for real-time multimedia services in LTE networks", IEEE Transactions on Multimedia, vol. 13, no. 5, (2011), pp. 1052-1065.

[7] 3GPP TS 36.101, "User Equipment (UE) radio transmission and reception,

[8] A. Ghosh, "LTE-advanced: next-generation wireless broadband technology [Invited Paper]", IEEE Wireless Communications, vol. 17, no. 3, (2010).

[9] S. Suganya, and C. Ramesh, "SNR based Predictive Packet Scheduling in LTE with Varied CQI", Indian Journal of Science and Technology, vol. 9, no. 47, (2016). 
[10] Y. X. Wang, "Packet Scheduling in LTE with Imperfect CQI.", International Journal of Advanced Research in Computer Science and Software Engineering, vol. 3, no. 6, (2013), pp. 6-13.

[11] G. Wang, "Power scheduling of Kalman filtering in wireless sensor networks with data packet drops", arXiv preprint arXiv:1312.3269, (2013).

[12] W. K. Lai and C.-L. Tang, "QoS-aware downlink packet scheduling for LTE networks", Computer Networks, vol. 57, no. 7, (2013), pp. 1689-1698

[13] M. Kalil, A. Shami and A. Al-Dweik, "QoS-aware power-efficient scheduler for LTE uplink", IEEE Transactions on Mobile Computing, vol. 14, no. 8, (2015), pp. 1672-1685.

[14] R. E. Kalman, "A new approach to linear filtering and prediction problems", Journal of basic Engineering, vol. 82, no. 1, (1960), pp. 35-45.

[15] K. You, N. Xiao and L. Xie, "Kalman filtering with scheduled measurements", Analysis and Design of Networked Control Systems. Springer London, (2015), pp. 269-291. 